\title{
Possibilities of high precision GPS data in autonomous driving
}

\author{
Márton Pál*, Fanni Vörös*, István Elek, Béla Kovács \\ ELTE Eötvös Loránd University, Department of Cartography and Geoinformatics, marchello@map.elte.hu, \\ vorosfanni@map.elte.hu,elek@map.elte.hu,climbela@map.elte.hu
}

Keywords: GPS, autonomous driving, self-driving car, high precision, database

\begin{abstract}
:
A self-driving car is a vehicle that is able to perceive its surroundings and navigate in it without human action. Radar sensors, lasers, computer vision and GPS technologies help it to drive individually (Figure 1). They interpret the sensed information to calculate routes and navigate between obstacles and traffic elements.
\end{abstract}

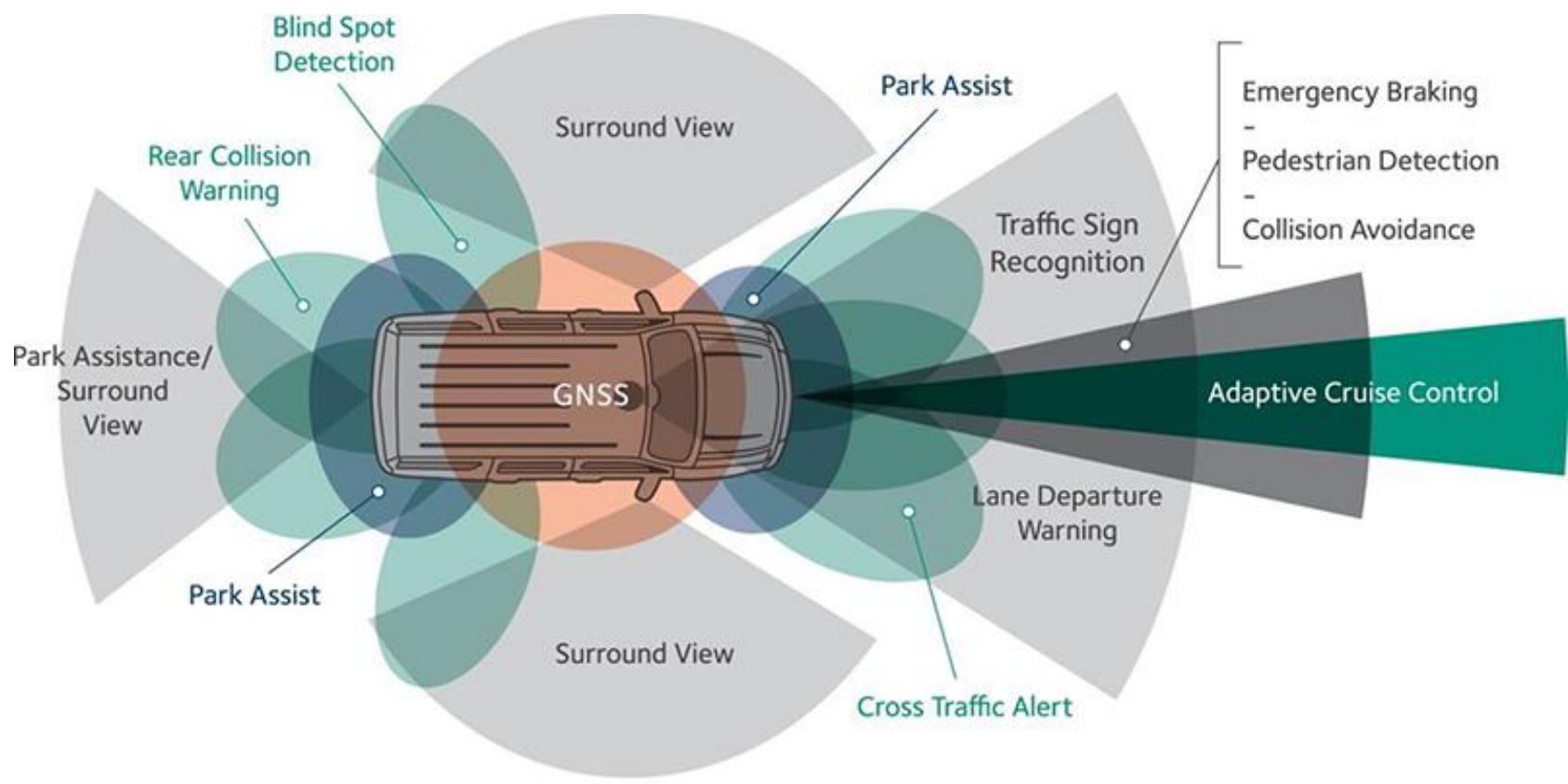

\section{Long-Range Radar Short/Medium Range Radar}

LIDAR

Camera

Ultrasound

GNSS

Figure 1. The sensors of a self-driving car (source: novatel.com)

Sufficiently accurate navigation and information about the current position of the vehicle are indispensable for transport. These expectations are fulfilled in the case of a human driver: the knowledge on traffic rules and signs make possible to navigate through even difficult situations. Self-driving systems substitute humans by monitoring and evaluating the surrounding environment and its objects without the background information of the driver. This analysing process is vulnerable. Sudden or unexpected situations may occur but high precision navigation and background GPS databases can complement sensor-detected data.

The assistance of global navigation has been used in cars for decades. Drivers can easily plan their routes and reach their destination by using car GPS units. However, these devices do not provide accurate positioning: there may be a difference of several metres from the real location. Self-driving cars also use navigation to complement sensor data. Although there are already autonomous system tests on motorways and countryside roads, in densely built-in areas this technology faces complications due to accuracy problems. The dilution of precision (DOP) values can be extremely high in larger settlements because high buildings may hide southern sky (where satellite signs are sensed from on our latitude).

We can achieve centimetre-level accuracy (if the conditions are ideal) with geodesic RTK (real-time kinematic) GPS systems. This high-precision position data is derived from satellite-based positioning systems. Measurements of the phase of the signal's carrier wave are real-time corrected by a single reference or an interpolated virtual station. 
In this research we use RTK GPS technology in order to work out a spatial database. These measurements can also be less precise in dense cities, but there is time during fieldwork to try to eliminate inaccuracy. We have chosen a sample area in the inner city of Budapest, Hungary where we located all traffic signs, pedestrian crossings and other important elements. As self-driving cars need precise position data of these terrain objects, we have tried to work with a maximum error of a few decimetres.

We have examined online map providers if they have feasible data structure and some base data. The implemented structure is similar to OpenStreetMap DB, in which there are already some traffic lights in important crossings. With this preliminary test database, we would like to filter out dangerous situations. If the camera of the car does not see a traffic sign because of a tree or a truck, information about it will be available from the database. If a pedestrian crossing is hardly visible and the sensor does not recognize it, the background GIS data will warn the car that there may be inattentive people on the road.

A test application has also been developed (Figure 2.), in which our Postgres/Postgis database records have been inserted. In the next phase of the project we try to test our database in the traffic. We plan to drive through the sample area and observe the GPS accuracy in the recognition of the located signs.

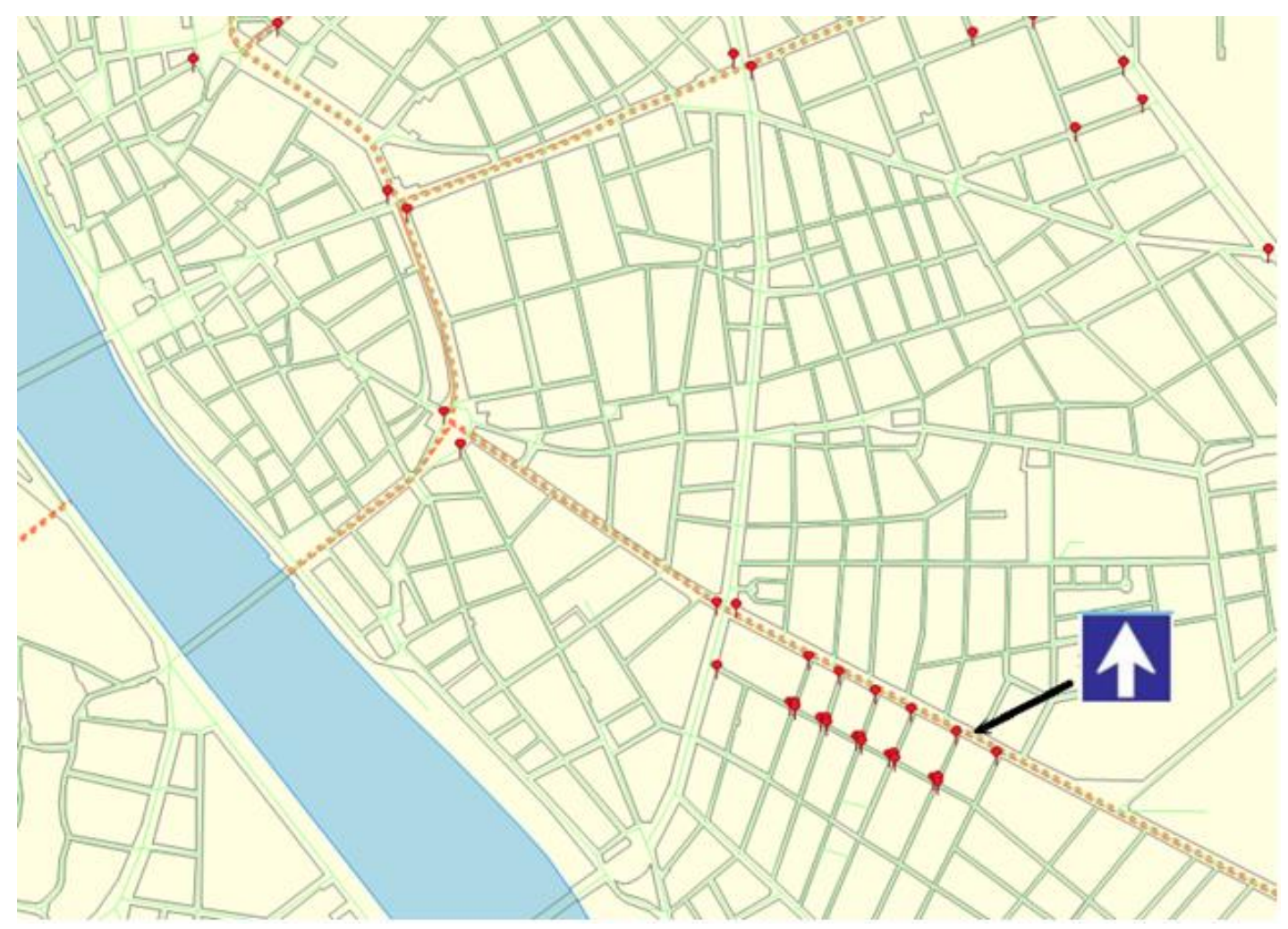

Figure 2. The test application showing some traffic signs

This research aims to achieve higher safety in the field of autonomous driving. By having a refreshable cartographic GIS database in the memory of a self-driving car, there is a smaller chance of risking human life. However, the maintenance demands a high amount of work. Because of this we should concentrate only on the most important signs. Even the cars can be able to supervise the content of the database if there is a large number of them on the road. The frequent production and analysis of point clouds is also an option to get nearer to safe automatized traffic.

EFOP-3.6.3-VEKOP-16-2017-00001: Talent Management in Autonomous Vehicle Control Technologies - The Project is supported by the Hungarian Government and co-financed by the European Social Fund. 